\title{
Postoperative Evaluation of Knees With Posterior Cruciate Ligament Injury Using Patient-Oriented Evaluation Method: Comparison of Single-Bundle and Anatomic Double-Bundle Techniques
}

Satoshi Ochiai ( $\nabla$ hxcmk230@ybb.ne.jp )

NHO Kofu National Hospital https://orcid.org/0000-0001-7202-6101

Tetsuo Hagino

NHO Kofu National Hospital

Shinya Senga

NHO Kofu National Hospital

Naoto Furuya

Yamanashi Daigaku

Naofumi Taniguchi

Yamanashi Daigaku

Takashi Ando

Yamanashi Daigaku

Hirotaka Haro

Yamanashi Daigaku

\section{Research Article}

Keywords: Posterior cruciate ligament, Reconstruction, Double bundle, SF-36, Treatment outcome

Posted Date: October 27th, 2021

DOI: https://doi.org/10.21203/rs.3.rs-961627/v1

License: (c) (1) This work is licensed under a Creative Commons Attribution 4.0 International License. Read Full License 


\section{Abstract}

Introduction Using the patient-based QOL evaluation scale SF-36 and conventional assessment methods, we evaluated the postoperative outcome of patients with posterior cruciate ligament (PCL) injury who underwent single-bundle or double-bundle reconstruction, and compared the two reconstruction techniques.

Methods 37 male patients with isolated PCL injury who underwent reconstruction were randomized to receive single-bundle reconstruction (group $S: n=20$ ) or double-bundle reconstruction (group $D: n=17$ ). Before surgery and 6 and 24 months after surgery, patients were evaluated by SF-36 scores, Lysholm score, visual analog scale (VAS), posterior tibial displacement rate, and knee range of motion (ROM).

Results For SF-36 evaluation at 6 months post-surgery, the scores of all the subscales improved to above the national standard values in group $D$, whereas none of the subscale scores reached the national standard values in group $S$, and three subscale scores were inferior in group $S$ compared to group $D$. At 24 months post-surgery, improvement of all subscale scores to above the national standard values was achieved in both groups. Lysholm score, VAS score, and posterior tibial displacement rate improved after surgery in both groups, but no significant intergroup differences were observed in all evaluation methods. For knee ROM, residual limitation of flexion was significantly more frequent in group $S$ than in group $D$ at 6 and 24 months post-surgery.

Conclusion Arthroscopy-assisted single-bundle PCL reconstruction technique is considered to be a safe procedure with low invasiveness, but despite its widespread use, surgical result is not consistently good. This was attributed to the low reproducibility of the unique course and anatomy of PCL, and the anatomic double-bundle reconstruction technique was proposed aiming to improve treatment outcome. According to the present results, double-bundle reconstruction tended to achieve better restoration at an early stage compared to single-bundle reconstruction, with fewer patients having residual limitation of knee flexion after surgery.

Trial registration number of our hospital's IRB: 27-8.

Registered 14 September 2015, retrospectively registered.

\section{Introduction}

The posterior cruciate ligament (PCL) is the thickest and strongest ligament in the knee joint and is responsible for $85-100 \%$ of posterior stabilization of the knee joint $[1,2]$. This ligament is composed of two bundles of fibers: the anterolateral bundle (ALB) that tightens in knee flexion, and the posteromedial bundle (PMB) that tightens in knee extension. The posterior stabilizing effect is obtained by the codominant relationship of these two bundles $[3,4]$. 
Ligament reconstruction is considered the first choice of surgical treatment for PCL injury, and a modality that regains knee function at high activity level. In recent years, the double-bundle reconstruction method that allows more precise and anatomic reproduction of the PCL has been developed $[5,6]$. Both basic and clinical studies have reported the superiority of the double-bundle reconstruction technique over the conventional single-bundle reconstruction method $[7,8]$. On the other hand, there are also reports that showed no clear difference in treatment outcome between the single-bundle and double-bundle reconstruction techniques [9-11], or even when subjective evaluation showed superiority of the doublebundle reconstruction method, no significant difference in clinical outcome was found between the two techniques [12-14].

We have evaluated patients with knee ligament injuries using the Medical Outcome Study 36 -item shortform health survey (SF-36) $[15,16]$ that allows patient-oriented assessment of QOL subdivided into several health domains, and reported the importance of using not only doctor-based objective assessments but also patient-based subjective evaluation [17-20]. In the present study, we recruited patients who underwent anatomic double-bundle or single-bundle PCL reconstruction, and evaluated them over time by conventional assessment methods as well as SF-36, and compared the treatment outcome of the two surgical techniques.

\section{Patients And Methods}

\section{Subjects and protocol}

Of 113 men who were diagnosed with PCL injury at presentation to the Sports Medicine and Knee Center of Kofu National Hospital between January 2007 and February 2018, 94 patients with isolated PCL injury were followed prospectively, after excluding 19 patients with concurrent injuries of other ligaments, or severe meniscal or articular cartilage injuries. Of the 94 patients followed, 37 who opted for PCL reconstruction after continuously complaining of subjective symptoms for more than 3 months after injury, and underwent primary PCL reconstruction using autologous flexor tendons were included in the present study. The patients were randomly assigned to receive one of two reconstruction techniques: 20 patients (aged 15 to 47 years, mean age 26.2 years) underwent single-bundle reconstruction (group S) and 17 patients (aged 16 to 52 years, mean age 29.4 years) underwent double-bundle reconstruction (group D). The subjects were followed until 24 months after surgery (Figure 1). Only men were included as subjects in the present study, because double-bundle reconstruction was difficult to perform in women with small physical stature.

\section{Outcome measures}

In all patients, QOL was evaluated by SF-36 before surgery and 6 and 24 months after surgery, and the results were compared with the Japanese national standard (NBS; norm-based scoring: absolute scores of 0-100 were recalculated by standardizing each scale to have a mean score of 50 and standard deviation of 10 in the general Japanese population). In addition, the knee function in the same periods was assessed using the Visual Analog Scale (VAS, a clinical pain scale) [21], Lysholm scoring scale 
(minimum score 0, maximum score 100; scores below 65 are interpreted as poor function) [22], posterior tibial displacement rate measured from a stress plain radiograph taken while using a Telos SE device (Telos Japan, Tokyo, Japan) (Measurement was made with the knee flexed to $90^{\circ}$ and a force of $15 \mathrm{KPa}$ applied to the anterior aspect of the center of tibia. Displacement was measured as the mid-point displacement rate. PCL impairment was diagnosed when the displacement rate was $45 \%$ or below) [2325], and the difference in range of motion between the affected and unaffected knees. These outcome measures were compared between group $S$ and group $D$.

SF-36 is composed of the following 8 subscales: physical functioning (PF) role- physical (RP), bodily pain (BP) and general health (GH), which constitute the physical health component; as well as vitality $(\mathrm{VT})$, social functioning (SF), role-emotional (RE) and mental health $(\mathrm{MH})$, which constitute the mental health component.

\section{Surgical techniques}

All the surgeries were performed by the first author (S.O.) as the main operator.

To harvest and prepare the graft tendon, the patient was placed supine with the knee joint flexed at approximately $90^{\circ}$. Through a skin incision of approximately $2.5 \mathrm{~cm}$ made on the medial side of the tibial tubercle, the semitendinosus tendon was elevated, together with the gracilis tendon if needed. Then the harvested tendon was bundled, and the two ends were attached to artificial tendons (Endobutton Tape and Endobutton CL; Acufex; Smith \& Nephew Mansfield, Massachusetts) to prepare the tendon graft. An arthroscope with $30^{\circ}$ oblique view was used, and arthroscopic procedures were conducted via the anteromedial, antero-lateral and postero-medial portals.

\section{Single-bundle reconstruction}

Using the Pro-trac PCL Guide System (Acufex; Smith \& Nephew), a specialized guide wire was inserted into the center of the PCL tibial footprint, and a cannulated drill and dilator were used to create a bone tibial tunnel with a diameter determined according to the width of the tendon graft. Next, using a Flip Cutter II (Arthrex), a bone femoral tunnel with a diameter depending on the width of the tendon graft was created by the outside-in method on the femoral side approximately $7 \mathrm{~mm}$ posterior to the margin of the articular cartilage at approximately 2 o'clock (right knee) or 10 o'clock (left knee) position. The tendon graft on the femoral side was fixed using Endobutton (Acufex) and that on the tibial side using spike staples by double stapling method. The graft was fixed at $90^{\circ}$ knee flexion with a tension of $60 \mathrm{~N}$ applied to the tendon graft. All the tendon grafts prepared had diameters of $8.5 \mathrm{~mm}$ or larger $(8.5$ to $9.0 \mathrm{~mm})$ and lengths of $65 \mathrm{~mm}$ or longer ( 65 to $75 \mathrm{~mm}$ ).

\section{Double-bundle reconstruction}

Using the Pro-trac ACL Guide System, a guide wire was inserted into the posteromedial part of the PCL tibial footprint, and then a cannulated drill and dilator were used to drill a bone tibial tunnel with the same diameter as the PMB of the graft tendon. Using the same method, another bone tibial tunnel with the same diameter as the ALB of the tendon graft was made in the anterolateral part of the PCL tibial 
footprint. Next, two femoral tunnels, each with the same diameter as the PMB or ALB, were created using the outside-in method. The tunnel for PMB reconstruction was located approximately $8 \mathrm{~mm}$ posterior to the articular cartilage margin at the anterior aspect of the intercondylar fossa at approximately 2:30 o'clock (right knee) or 10:30 o'clock (left knee) position. The tunnel for ALB reconstruction was located approximately $6 \mathrm{~mm}$ from the articular cartilage margin at approximately 1:00 o'clock (right knee) or 11:00 o'clock (left knee) position. For both bundles, the femoral side was fixed with Endobutton and the tibial side with spike staples by double stapling method. The bundles were fixed at $90^{\circ}$ knee flexion for the AMB and full knee extension for the PLB, while applying a tension of $40 \mathrm{~N}$ to the tendon graft. In all the tendon grafts harvested, both bundles had diameters of $6 \mathrm{~mm}$ or larger $(6-7 \mathrm{~mm})$ and lengths of 60 $\mathrm{mm}$ or longer $(60-70 \mathrm{~mm})$.

\section{Postoperative management}

The postoperative management protocol was the same for group $S$ and group $D$. Range of motion training while wearing an orthosis with angle limitation was started from 2 weeks after surgery. Partial weight-bearing was permitted from 3 weeks, and full weight bearing from 6 weeks. Sports activities were restarted from around 9 months after surgery.

\section{Statistical analysis}

Mann-Whitney's $U$ test and two-way ANOVA were used for statistical analyses of data. A p value less than 0.05 was considered significant. Statistics analyses were conducted using BellCurve for Excel (Social Survey Research Information Co., Ltd.).

\section{Results}

No serious postoperative complications such as re-rupture and deep wound infection occurred in both group $S$ and group $D$, and none of the patients required re-operation. None of the patients deviated from the protocol after surgery.

\section{Subjective evaluation by SF-36}

The results of evaluation using SF-36 are shown in Figure 2. At 6 months after surgery, the scores of all the subscales improved to above the national standard values in group $D$, whereas none of the subscale scores reached the national standard values in group S. Furthermore, PF, RP and BP scores in group S were significantly worse than those in group $D(p<0.05)$.

At 24 months after surgery, both groups $S$ and $D$ achieved improvement of all subscale scores to above the national standard values. Moreover, PF, RP and BP scores improved significantly compared to before surgery in both groups $(p<0.05)$.

\section{Evaluation by Lysholm score}


The mean Lysholm scores before surgery and 6 and 24 months after surgery were respectively $47.2 \pm$ $21.3,79.3 \pm 18.8$ and $80.6 \pm 20.5$ in group S; and $51.9 \pm 22.2,90.3 \pm 12.1$ and $90.6 \pm 8.8$ in group D. Significant improvement was observed at 6 and 24 months after surgery compared to before surgery in both group $S$ and group $D$, but no significant intergroup differences were found.

\section{Evaluation of pain by VAS}

The mean VAS scores before surgery and 6 and 24 months after surgery were respectively $45.5 \pm 25.8$, $19.4 \pm 20.3$ and $16.1 \pm 23.5$ in group $S$; and $54.5 \pm 24.4,16.4 \pm 19.2$ and $11.8 \pm 15.5$ in group D. Although significant improvement was achieved at 6 and 24 months after surgery compared to before surgery in both groups, there were no significant differences between the two groups.

\section{Evaluation of knee instability by posterior tibial displacement rate}

The mean posterior tibial displacement rates (\%) before surgery and 6 and 24 months after surgery were respectively $44.6 \pm 9.1,51 \pm 7.7$ and $53.7 \pm 6.8$ in group $S$; and $43.9 \pm 4.2,52.3 \pm 7$ and $51.6 \pm 5.9$ in group D. Although significant improvement was obtained after surgery compared to before surgery in both groups, no significant intergroup differences were observed.

\section{Evaluation of knee range of motion}

The results of knee range of motion are shown in Table 1. Limitation of flexion of 5 degree or more remained detectable at 6 and 24 months after surgery in 9 patients (45\%) and 6 patients (30\%), respectively, in group S; and in 2 patients (30\%) and 1 patient (5\%) in group D. The proportions of patients with limitation of flexion were significantly higher in group $S$ than in group $D$ ( 6 months after surgery: $p=$ $0.021,24$ months after surgery: $p=0.041$ ).

Table 1

Evaluation of knee range of motion.

\begin{tabular}{|lllll|}
\hline & & Group S & Group D & p \\
\hline Flexion & $\mathbf{6}$ months & $9 / 20(45 \%)$ & $2 / 17(12 \%)$ & 0.021 \\
\hline Extension & 24 months & $6 / 20(30 \%)$ & $1 / 17(5.9 \%)$ & 0.041 \\
\hline & 6 months & $2 / 20(10 \%)$ & $1 / 17(5.9 \%)$ & n.s. \\
\hline $\begin{array}{l}\text { Group S: single-bundle posterior cruciate ligament (PCL) reconstruction. Group D: double-bundle PCL } \\
\text { reconstruction. Data are expressed as number of patients with limitation of flexion } \geq 5 \text { degree/total } \\
\text { number of patients, with percentage in parenthesis. }\end{array}$ \\
\hline
\end{tabular}

\section{Discussion}


PCL has a strong innate healing capacity, and many patients with PCL injury attain good improvement with conservative therapy [26-29], but surgery is selected by patients in whom severe posterior instability remains and subjective symptoms persist [30]. Among the surgical modalities, arthroscopic single-bundle PCL reconstruction is widely used in view of its low invasiveness and safety. However, according to a systematic review reported by Kim et al. [31], arthroscopically assisted single-bundle PCL reconstruction for high-grade PCL injuries provides some improvement of instability, but does not restore normal knee stability or prevent the development of degenerative osteoarthritis. In our previous studies, we found that persistent limitation of flexion accompanied by pain deteriorated the treatment result of single-bundle PCL reconstruction $[17,18]$.

Low reproducibility of the unique course and anatomy of the PCL was considered to be the cause of unsuccessful PCL reconstruction [32]. To overcome these issues, the anatomic double-bundle reconstruction method was developed $[5,6]$. In this study, we compared the relative merits and demerits of the single-bundle and double-bundle reconstruction techniques using the patient-based SF-36 healthrelated QOL scale with scientifically proven reliability and validity $[33,34]$ together with the conventional objective clinical measures.

In the present study, improvements in Lysholm score, VAS score, and posterior tibial displacement rate after surgery compared to before surgery were achieved by both single-bundle and double-bundle reconstruction techniques, with no significant intergroup differences in all three objective assessment methods. On the other hand, evaluation using SF-36 showed improvement of all subscale scores to above the national standard values in group $D$ from the early post-surgical period of 6 months, whereas none of the subscale scores reached the national standard values in group $S$, and significant intergroup differences in three subscales belonging to the physical health component were observed. At 24 months after surgery, improvement of all subscale scores to above the national standard was attained in both groups, and all the subscale scores were apparently higher in group $D$ than in group $S$, although there were no significant differences. Regarding range of motion of the knee, significantly higher proportions of knees in group $S$ had residual limitation of flexion compared to group D, both at 6 and 24 months after surgery.

By reconstructing the ALB and PMB separately, the double-bundle reconstruction technique is considered capable of mimicking the native PCL both anatomically and functionally [7, 35-37]. We speculate that in the double-bundle reconstruction, the morphology of the tendon graft divided into two bundles more closely reproduces the flat structure of the native PCL and reduces the interference in the popliteal region during flexion, which may have decreased the limitation of flexion after reconstruction as observed in this study. Smooth knee motion relieves the physical pain from the early period after surgical, which probably contributes to favorable subjective evaluation of the double-bundle reconstruction technique by patients.

At the last evaluation of treatment outcome, overall improvement was observed in both subjective and objective evaluations for both surgical techniques, with no clear differences. However, we believe that 
anatomic double-bundle reconstruction, which confers benefits of smooth knee motion early after surgery and low rate of residual limitation of flexion, should be recommended.

\section{Abbreviations}

PCL: posterior cruciate ligament ; ALB: anterolateral bundle ; PLB: posteromedial bundle; SF-36: 36-item short-form health survey; NBS: norm-based scoring; VAS: visual analog scale; ROM: range of motion; PF: physical functioning; RP: role- physical; BP: bodily pain; GH: general health; VT: vitality; SF: social functioning; RE: role-emotional; $\mathrm{MH}$ : mental health

\section{Declarations}

\section{Ethical approval and consent to participate}

We carried out a prospective clinical trial Ethical approval was obtained from the institutional review board of the National Hospital Organization, Kofu National Hospital. Informed consent was obtained from all the patients.

\section{Consent for publication}

Not applicable

\section{Availability of data and materials}

All data and materials were in the compliance with the journal's policy fully.

\section{Competing interests}

The authors declare that they have no competing interests.

\section{Funding}

None

\section{Author's contributions}

SO designed and wrote the manuscript. SS, NF, NT and TA collected and analyzed research data. TH and $\mathrm{HH}$ interpreted research data and edit the manuscript. All authors read and approved the final manuscript.

\section{References}

1. Johnson JC, Bach BR. Current concepts review, posterior cruciate ligament. Am J Surg. 1990;3:14353. 
2. Fu FH, Harner CD, Johnson DL, Miller MD, Woo SLY. (1993) Biomechanics of knee ligaments: basic concepts and clinical application. J Bone Joint Surg [Am];75-A:1716-27.

3. Chahla J, Moatshe G, Engebretsen L, LaPrade RF. Anatomic double-bundle posterior cruciate ligament reconstruction. JBJS Essent Surg Tech. 2017;7(1):e4. doi:10.2106/JBJS.ST.16.00083.

4. Kennedy NI, Wijdicks CA, Goldsmith MT, et al. Kinematic analysis of the posterior cruciate ligament, part 1: the individual and collective function of the anterolateral and posteromedial bundles. Am J Sports Med. 2013;41(12):2828-38.

5. Makino A, Aponte Tinao L, Ayerza MA, et al. Anatomic double-bundle posterior cruciate ligament reconstruction using double-double tunnel with tibial anterior and posterior fresh-frozen allograft. Arthroscopy. 2006;22(6):684.e1-5.

6. Race A, Amis AA. PCL reconstruction. In vitro biomechanical comparison of 'isometric' versus single and double-bundled 'anatomic' grafts. J Bone Joint Surg Br. 1998;80(1):173-9. doi:10.1302/0301$620 x .80 \mathrm{~b} 1.7453$.

7. Amis AA, Gupte CM, Bull AM, Edwards A. Anatomy of the posterior cruciate ligament and the meniscofemoral ligaments. Knee Surg Sports Traumatol Arthrosc. 2006;14(3):257-63. doi:10.1007/s00167-005-0686-x.

8. Milles JL, Nuelle CW, Pfeiffer F, Stannard JP, Smith P, Kfuri M Jr, Cook J. Biomechanical comparison: single-bundle versus double-bundle posterior cruciate ligament reconstruction techniques. J Knee Surg. 2017;30(4):347-51. doi:10.1055/s-0036-1588014.

9. Qi YS, Wang HJ, Wang SJ, Zhang ZZ, Huang AB, Yu JK. A systematic review of double-bundle versus single-bundle posterior cruciate ligament reconstruction. BMC Musculoskelet Disord. 2016;17:45. doi:10.1186/s12891-016-0896-z. PMID: 26818255; PMCID: PMC4730768.

10. Kohen RB, Sekiya JK. Single-bundle versus double-bundle posterior cruciate ligament reconstruction. Arthroscopy. 2009;25(12):1470-7. doi:10.1016/j.arthro.2008.11.006.

11. Deie M, Adachi N, Nakamae A, Takazawa K, Ochi M. (2015) Evaluation of single-bundle versus double-bundle PCL reconstructions with more than 10-year follow-up.

ScientificWorldJournal.;2015:751465. doi: 10.1155/2015/751465.

12. Yoon KH, Bae DK, Song SJ, Cho HJ, Lee JH. A prospective randomized study comparing arthroscopic single-bundle and double-bundle posterior cruciate ligament reconstructions preserving remnant fibers. Am J Sports Med. 2011;39(3):474-80. doi:10.1177/0363546510382206. Epub 2010 Nov 23.

13. Li Y, Li J, Wang J, Gao S, Zhang Y. Comparison of single-bundle and double-bundle isolated posterior cruciate ligament reconstruction with allograft: a prospective, randomized study. Arthroscopy. 2014;30(6):695-700.

14. Jain V, Goyal A, Mohindra M, Kumar R, Joshi D, Chaudhary D. A comparative analysis of arthroscopic double-bundle versus single-bundle posterior cruciate ligament reconstruction using hamstring tendon autograft. Arch Orthop Trauma Surg. 2016;136(11):1555-61. doi:10.1007/s00402-016-2512y. 
15. Fukuhara S, Suzukamo Y. (2004) Manual of SF-36v2 Japanese version. Kyoto, Japan: Institute for health Outcomes \& Process Evaluation Research. 2004.

16. Fukuhara S, Ware JE Jr, Kosinski M. Psychometric and clinical tests of validity of the Japanese SF36 health survey. J Clin Epidemiol. 1998;51:1045-53.

17. Ochiai S, Hagino T, Senga S, Yamashita T, Ando T, Haro H. Prospective analysis using a patientbased health-related scale shows lower functional scores after posterior cruciate ligament reconstructions as compared with anterior cruciate ligament reconstructions of the knee. Int Orthop. 2016;40(9):1891-8. doi:10.1007/s00264-016-3189-0.

18. Ochiai S, Hagino T, Senga S, Yamashita T, Haro H. Treatment outcome of reconstruction for isolated posterior cruciate injury: subjective and objective evaluations. J Knee Surg. 2019;32(6):506-12. doi:10.1055/s-0038-1653947.

19. Ochiai S, Hagino T, Senga S, Saito M, Haro H. Prospective evaluation of patients with anterior cruciate ligament reconstruction using a patient-based health-related survey: comparison of singlebundle and anatomic double-bundle techniques. Arch Orthop Trauma Surg. 2012;132(3):393-8. doi:10.1007/s00402-011-1443-x.

20. Ochiai $\mathrm{S}$, Hagino $\mathrm{T}$, Tonotsuka $\mathrm{H}, \mathrm{Haro} \mathrm{H}$. Health-related quality of life in patients with an anterior cruciate ligament injury. Arch Orthop Trauma Surg. 2010;130(3):397-9. doi:10.1007/s00402-0090964-z.

21. Aitken RCB. (1969) Measurement of feelings using visual analogue scales. Proceedings of the Royal Society of Medicine;62,989-93.

22. Tegner Y, Lysholm J. Rating systems in the evaluation of knee ligaments injuries. Clin Orthop Relat Res. 1985;198:43-9.

23. Murase K, Kumano K, Mannouji T, Yokoe S, Kaneko K, Irie K, Okubo F. Radiographical measurement of anteroposterior instability of the knee joint. Japanese: Tokyo Knee Joint Meeting for Study; 1987. pp. 179-86.

24. Maruyama Y, Shitoto K, Baba T, Kaneko K. Evaluation of the clinical results of posterior cruciate ligament reconstruction -a comparison between the use of the bone tendon bone and semitendinosus and gracilis tendons-. Sports Med Arthrosc Rehabil Ther Technol. 2012;4(1):30. doi:10.1186/1758-2555-4-30.

25. Jung TM, Reinhardt C, Scheffler SU, Weiler A. (2006) Stress radiography to measure posterior cruciate ligament insufficiency: a comparison of five different techniques. Knee Surg Sports Traumatol Arthrosc;14 (11):1116-21.

26. Ahn JH, Lee SH, Choi SH, Wang JH, Jang SW. Evaluation of clinical and magnetic resonance imaging results after treatment with casting and bracing for the acutely injured posterior cruciate ligament. Arthroscopy. 2011;27(12):1679-87. doi:10.1016/j.arthro.2011.06.030.

27. Shelbourne KD, Davis TJ, Patel DV. The natural history of acute, isolated, nonoperatively treated posterior cruciate ligament injuries. A prospective study. Am J Sports Med. 1999;27(3):276-83. doi:10.1177/03635465990270030201. 
28. Jacobi M, Reischl N, Wahl P, Gautier E, Jakob RP. Acute isolated injury of the posterior cruciate ligament treated by a dynamic anterior drawer brace: a preliminary report. J Bone Joint Surg Br. 2010;92(10):1381-4. doi:10.1302/0301-620X.92B10.24807.

29. Agolley D, Gabr A, Benjamin-Laing H, Haddad FS. Successful return to sports in athletes following non-operative management of acute isolated posterior cruciate ligament injuries: medium-term follow-up. Bone Joint J. 2017;99-B(6):774-8. doi:10.1302/0301-620X.99B6.37953.

30. Winkler PW, Zsidai B, Wagala NN, Hughes JD, Horvath A, Senorski EH, Samuelsson K, MusahI V. Evolving evidence in the treatment of primary and recurrent posterior cruciate ligament injuries, part 2: surgical techniques, outcomes and rehabilitation. Knee Surg Sports Traumatol Arthrosc. 2021;29(3):682-93. doi:10.1007/s00167-020-06337-2.

31. Kim YM, Lee CA, Matava MJ. Clinical results of arthroscopic single-bundle transtibial posterior cruciate ligament reconstruction: a systematic review. Am J Sports Med. 2011;39(2):425-34. doi:10.1177/0363546510374452.

32. Fanelli GC, Giannotti BF, Edson CJ. (1994) The posterior cruciate ligament arthroscopic evaluation and treatment. Arthroscopy;10(6):673-88. Review.

33. Ware JE Jr, Sherbourne CD. The MOS 36-item short-form health survey (SF-36) I. Conceptual framework and item selection. Med Care. 1992;30(6):473-83.

34. Aaronson NK, Acquadro C, Fukuhara S, et al. International Quality of Life Assessment (IQOLA) Project. Qual Life Res. 1992;1(5):349-51.

35. Chahla J, Moatshe G, Cinque ME, Dornan GJ, Mitchell JJ, Ridley TJ, LaPrade RF. Single-bundle and double-bundle posterior cruciate ligament reconstructions: a systematic review and meta-analysis of 441 patients at a minimum 2 years' follow-up. Arthroscopy. 2017;33(11):2066-80. doi:10.1016/j.arthro.2017.06.049.

36. Wijdicks CA, Kennedy NI, Goldsmith MT, Devitt BM, Michalski MP, Årøen A, Engebretsen L, LaPrade RF. Kinematic analysis of the posterior cruciate ligament, part 2: a comparison of anatomic singleversus double-bundle reconstruction. Am J Sports Med. 2013;41(12):2839-48. doi:10.1177/0363546513504384.

37. Harner CD, Janaushek MA, Kanamori A, Yagi M, Vogrin TM, Woo SL. (2000) Biomechanical analysis of a double-bundle posterior cruciate ligament reconstruction. Am J Sports Med;28(2):144-51. doi: $10.1177 / 03635465000280020201$.

\section{Figures}




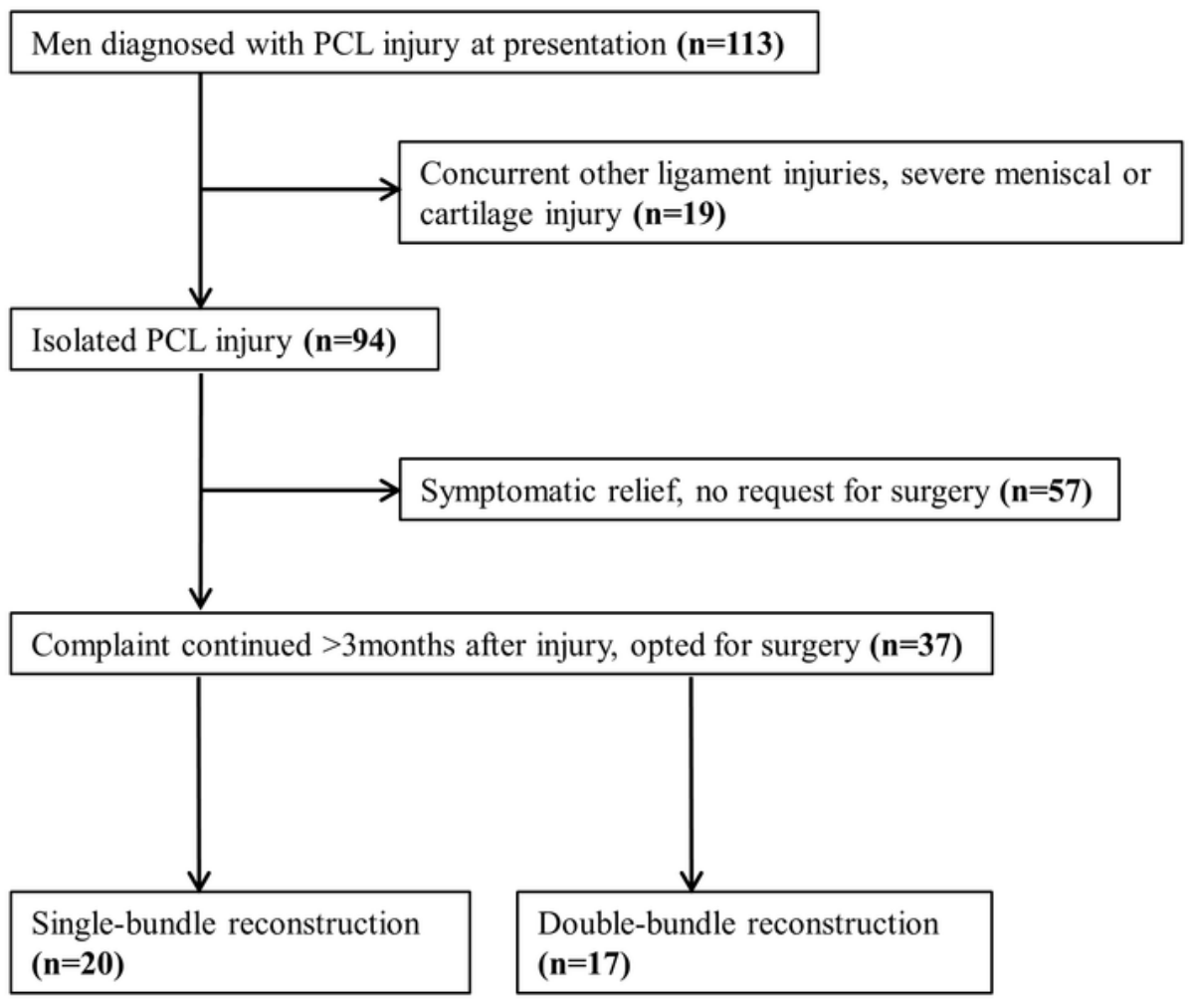

\section{Figure 1}

Flowchart of prospective study of male patients with posterior cruciate ligament injury treated at our center. 

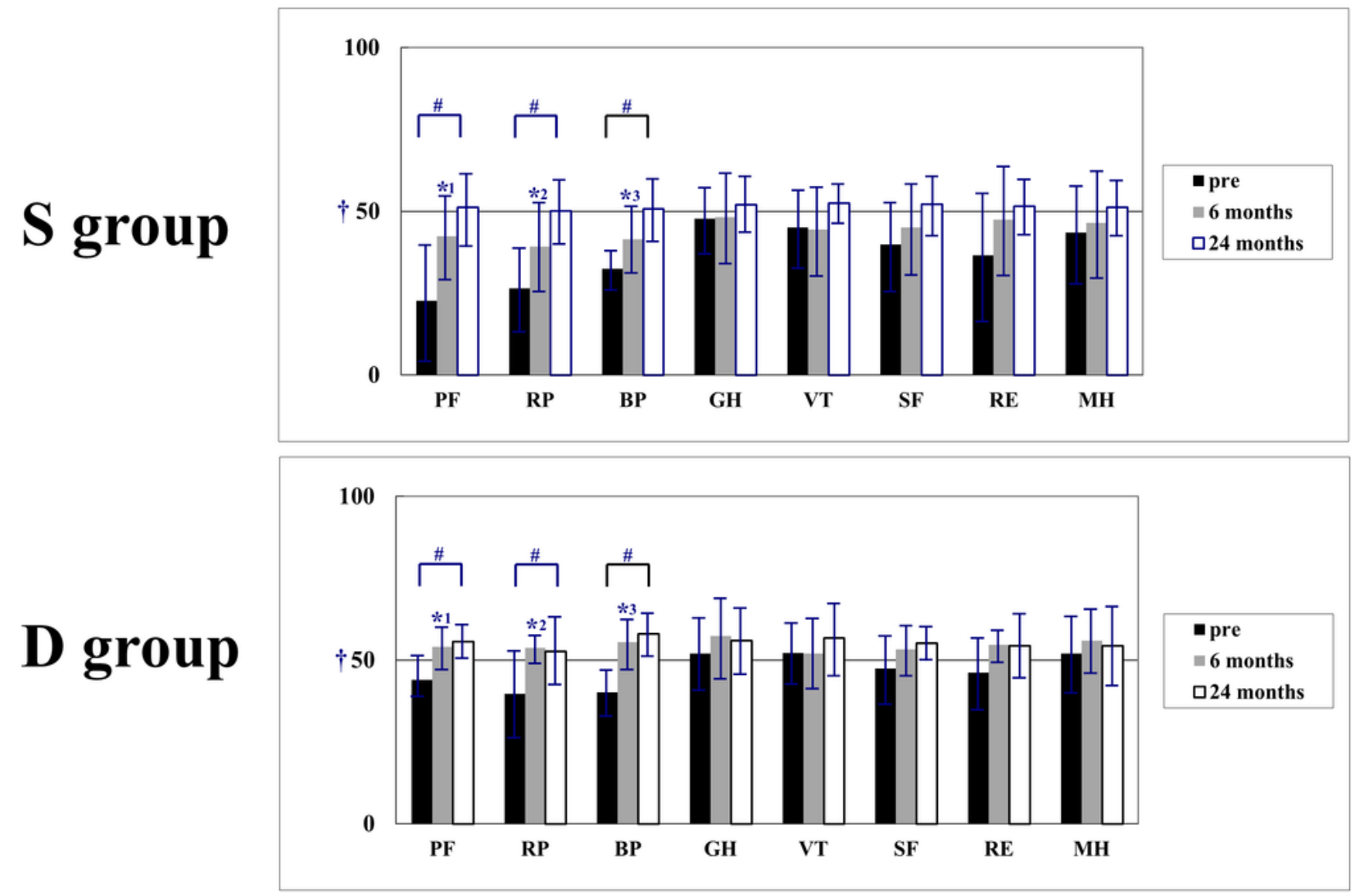

\section{Figure 2}

Results of subjective evaluation using SF-36. Group S: single-bundle posterior cruciate ligament (PCL) reconstruction. Group D: double-bundle PCL reconstruction. + : National standard values in Japan $=50$. ${ }^{*} p$ $<0.05$, group $S$ vs. group $D ; \# p<0.05,24$ months post-surgery vs. pre-surgery. At 6 months post-surgery, none of the subscale scores reached the national standard values in group $\mathrm{S}$, and significant differences in three subscales between group $S$ and group $D$ were observed. At 24 months post-surgery, improvement of all subscale scores to above the national standard values was achieved in both groups, and significant improvement of PF, RP and BP compared to pre-surgery scores was observed in both groups. 